\title{
Les micro-exploitations agricoles de plantes aromatiques et médicinales : élément marquant de l'agriculture urbaine à Ziguinchor, Sénégal
}

\author{
Maurice Dasylva $^{1, *}$, Ngor Ndour ${ }^{1}$, Bienvenu Sambou ${ }^{2}$ et Christophe Toussaint Soulard ${ }^{3}$ \\ ${ }^{1}$ Laboratoire d'agroforesterie et d'écologie (LAFE), Université Assane Seck de Ziguinchor (UASZ), BP 523, Ziguinchor, Sénégal \\ ${ }^{2}$ Université Cheikh Anta Diop, Institut des sciences de l'environnement, Dakar, Sénégal \\ ${ }^{3}$ UMR Innovation, Université Montpellier, INRA, Montpellier SupAgro, Montpellier, France
}

\begin{abstract}
Résumé - L'agriculture urbaine est une activité reconnue pour sa multifonctionnalité. En Afrique, elle peut contribuer à la sécurité alimentaire et à la réduction de la pauvreté. Souvent associée au maraîchage, et de plus en plus, à l'élevage urbain, la production de plantes aromatiques et médicinales est une forme d'agriculture urbaine peu étudiée. Elle est présente à Ziguinchor, au Sénégal, où a été menée l'étude. L'objectif de cet article est de caractériser cette agriculture et d'apprécier ses fonctions environnementales et socioéconomiques. La méthode combine une cartographie des sites agricoles et une enquête auprès de 70 producteurs. Les résultats montrent qu'il s'agit de micro-exploitations qui se développent dans les interstices urbains. La tenure foncière est dominée par l'emprunt gratuit à des propriétaires qui peuvent reprendre leurs terres à tout moment. Les espèces cultivées sont Mentha spicata L., Mentha X piperita L. et Ocimum basilicum L. La culture de ces plantes est l'œuvre des femmes qui en tirent des revenus significatifs. Ces revenus sont destinés à l'achat de denrées alimentaires et à la scolarisation des enfants. En plus de réduire les situations de pauvreté, ces micro-exploitations contribuent à l'assainissement de la ville, car la fertilisation provient des ordures ménagères organiques et de la fumure animale. Les menthes recèlent d'autres vertus, notamment médicinales, peu connues des producteurs. Malgré ces atouts, cette activité est limitée dans son développement alors que des solutions d'amélioration technique et de valorisation commerciale seraient possibles.
\end{abstract}

Mots clés : activité agricole / menthe / femme / alimentation / environnement

\begin{abstract}
Micro-farms producing aromatic and medicinal plants: a figure of the urban agriculture in Ziguinchor, Senegal. Urban agriculture is well known for its multifunctionality. In Africa, it can contribute to food security and poverty reduction. Often associated with urban gardening, and urban livestock, there is a gap of knowledge on the urban production of aromatic and medicinal plants. This production is present in Ziguinchor, Senegal, where the study has been conducted. The objective of this article is to describe this form of urban agriculture in order to evaluate its environmental and socioeconomic services. The method is based on mapping of agricultural sites in the city, and on a survey of 70 producers. The results show that these micro-farms are localized in urban interstices. The land tenure is based on free leases, from landowners who can take back their land at any time. The cultivated species are Mentha spicata L., Mentha $X$ piperita L. and Ocimum basilicum L. The cultivation of these plants is the work of women who get a significant income. These revenues are mainly intended for the purchase of food and the schooling of children. In addition to reducing poverty, these micro-farms contribute to the sanitation of the city, considering the fertilization coming from waste and animal manure. Mints have other properties, in particular medicinal, underestimated by the producers. In spite of these assets, this activity is limited in its development whereas solutions of technical improvement and commercial valorization would be possible.
\end{abstract}

Keywords: agricultural activity / mint / woman / food / environment

\footnotetext{
*Auteur de correspondance : dasylvamaurice@gmail.com
} 


\section{Introduction}

La croissance démographique des villes africaines entraîne leur extension sur les terres agricoles (Nouatin et Bachabi, 2010; Oladokoun, 2013). Parallèlement, l'agriculture urbaine est reconnue pour les services urbains qu'elle procure, au Nord comme au Sud (Hamilton et al., 2013; Mok et al., 2013). Son importance a été reconnue par la FAO (2010), qui affirme que 800 millions de citadins pratiquent l'agriculture urbaine dans le monde et que cette activité produit $15 \%$ des denrées alimentaires mondiales. Son rôle alimentaire est particulièrement fort en Afrique (De Bon et al., 2010) où elle fournirait $70 \%$ de la demande en légumes à Dakar et $90 \%$ à Accra $(\mathrm{Ba}$ et al., 2016); $60 \%$ de la demande en produits avicoles à Cotonou et $50 \%$ à Bamako (Akinbamijo et al., 2002). Plusieurs auteurs attestent, par ailleurs, que l'agriculture pratiquée en milieu urbain contribue à d'autres services, comme le recyclage des déchets urbains, la réduction de la pauvreté et l'édification de villes vertes (Smith et al., 2004; Mougeot, 2006; Ouédraogo, 2012; FAO, 2014).

Au Sénégal, la ville de Ziguinchor, qui comptait 218000 habitants en 2015, est peuplée à $92 \%$ de ménages agricoles (PAM, 2012), si l'on considère qu'un ménage regroupe des personnes vivant ensemble et mettant en commun tout ou partie de leurs ressources pour subvenir à leurs besoins primaires (Agence nationale de la statistique et de démographie [ANSD], 2014). Ziguinchor, située au sud-ouest du pays, présente un fort potentiel agricole compte tenu de son climat de type soudanien côtier Sud (Sagna, 2005), qui lui procure une température moyenne $\left(27,1^{\circ} \mathrm{C}\right)$ et une pluviométrie annuelle $(1316 \mathrm{~mm})$ favorables à une production agricole diversifiée et étalée dans l'année. Cependant, les espaces agricoles sont menacés par l'urbanisation.

La croissance démographique y est soutenue. Ziguinchor fut pendant plus de trente ans le foyer d'accueil de populations rurales fuyant le conflit casamançais (Robin, 2006). Ces dernières, arrivées en ville sans qualification professionnelle, ont continué à pratiquer l'agriculture pour assurer leurs moyens de subsistance. Plus récemment, la ville s'est étendue avec la création d'une université, une nouvelle emprise qui a accentué la pression foncière sur les terres proches. Une conséquence est la prolifération des habitats spontanés en périphérie de la ville, notamment dans les vallées non aedificandi qui bordent la ville à l'est et à l'ouest et qui accueillent la quasi-totalité des activités de riziculture pluviale et de maraîchage de contre-saison (Dasylva, 2013; Keita, 2013).

Dans ce contexte, des habitants ont développé une activité agricole pratiquée dans les interstices urbains. Il s'agit de micro-exploitations produisant et commercialisant des plantes aromatiques et médicinales (PAM): menthe verte, menthe poivrée et basilic principalement. Ces plantes ont en effet plusieurs fonctions. Outre leurs usages à des fins alimentaires (consommation de thé, boissons, sirops), les menthes sont utilisées dans le domaine médical. Elles sont réputées efficaces contre la fatigue et les troubles digestifs. Zhiri et Baudoux (2005) ont montré que des espèces de menthes ont aussi des propriétés répulsives contre les moustiques vecteurs du paludisme, mettant en lumière l'intérêt de ces cultures pour la lutte contre l'endémie malarienne en Afrique occidentale.

Cette activité agricole reste cependant mal connue, et par conséquent très peu considérée par les autorités municipales.
Cette étude se propose de contribuer à la connaissance des micro-exploitations des PAM et vise, spécifiquement, à caractériser leurs fonctions environnementales et socioéconomiques. Notre hypothèse est que ces micro-exploitations de PAM offrent des services urbains utiles à la fois pour les producteurs et pour la ville. Pour l'explorer, nous avons développé une approche pluridisciplinaire de l'agriculture urbaine, de type «système agri-urbain » (Robineau et Soulard, 2017), croisant une approche géographique des sites d'agriculture urbaine, une lecture agronomique des modes de production, et une appréciation socioéconomique des besoins d'existence des ménages. La notion de micro-exploitation résume cette lecture englobante d'une réalité qui concerne des surfaces cultivées particulièrement restreintes. Elle désigne l'unité d'activité agricole concernée par la production de PAM, unité que l'on peut décrire par ses surfaces cultivées et le mode de tenure foncière correspondant, par sa main d'œuvre et les pratiques de production et de commercialisation, et par sa contribution au fonctionnement du ménage.

Dans les parties suivantes, nous présenterons les méthodes mobilisées pour construire et mener l'enquête à Ziguinchor. Puis, nous exposerons les résultats décrivant les microexploitations de PAM, leurs caractéristiques et leurs fonctions pour les ménages concernés. Nous discuterons alors ces résultats au regard de notre hypothèse relative aux services urbains que procurent ou pourraient procurer les PAM à Ziguinchor.

\section{Matériel et méthodes}

L'étude a été essentiellement menée par des enquêtes agrosocioéconomiques croisées avec des approches cartographiques des espaces productifs. L'objectif est de caractériser les microexploitations de PAM et d'analyser leur multifonctionnalité.

\subsection{Le choix des quartiers}

La ville de Ziguinchor est organisée en 27 quartiers (ANSD, 2015). La figure 1 permet de rendre compte de son organisation spatiale, située le long du fleuve Casamance et bordée de part et d'autres de vallées inondables mises en culture.

Des missions d'observation effectuées à l'échelle de la ville ont permis de recenser les quartiers où les PAM sont produites. Ce recensement a été réalisé par repérage des parcelles cultivées en compagnie des acteurs de base. Les 5 quartiers recensés sont Kandialang Est, Kansahoudy, Kénya, ainsi que Néma 2 et Château d'eau qui sont des sous-quartiers de Néma. Ils ont tous été retenus pour la réalisation de l'étude. Pour repérer les micro-exploitations étudiées au sein de ces quartiers, la méthode appliquée a été l'échantillonnage non probabiliste, par jugement a priori (Dufour et Larivière, 2012; Kherri, 2014) des quartiers.

\subsection{L'enquête auprès des producteurs}

Les enquêtes ont été réalisées auprès des producteurs de PAM. Du fait de l'absence de base de données sur ces types de producteurs dans la ville (Dasylva, 2013), la collecte des données s'est appuyée sur le porte-à-porte. La procédure a 


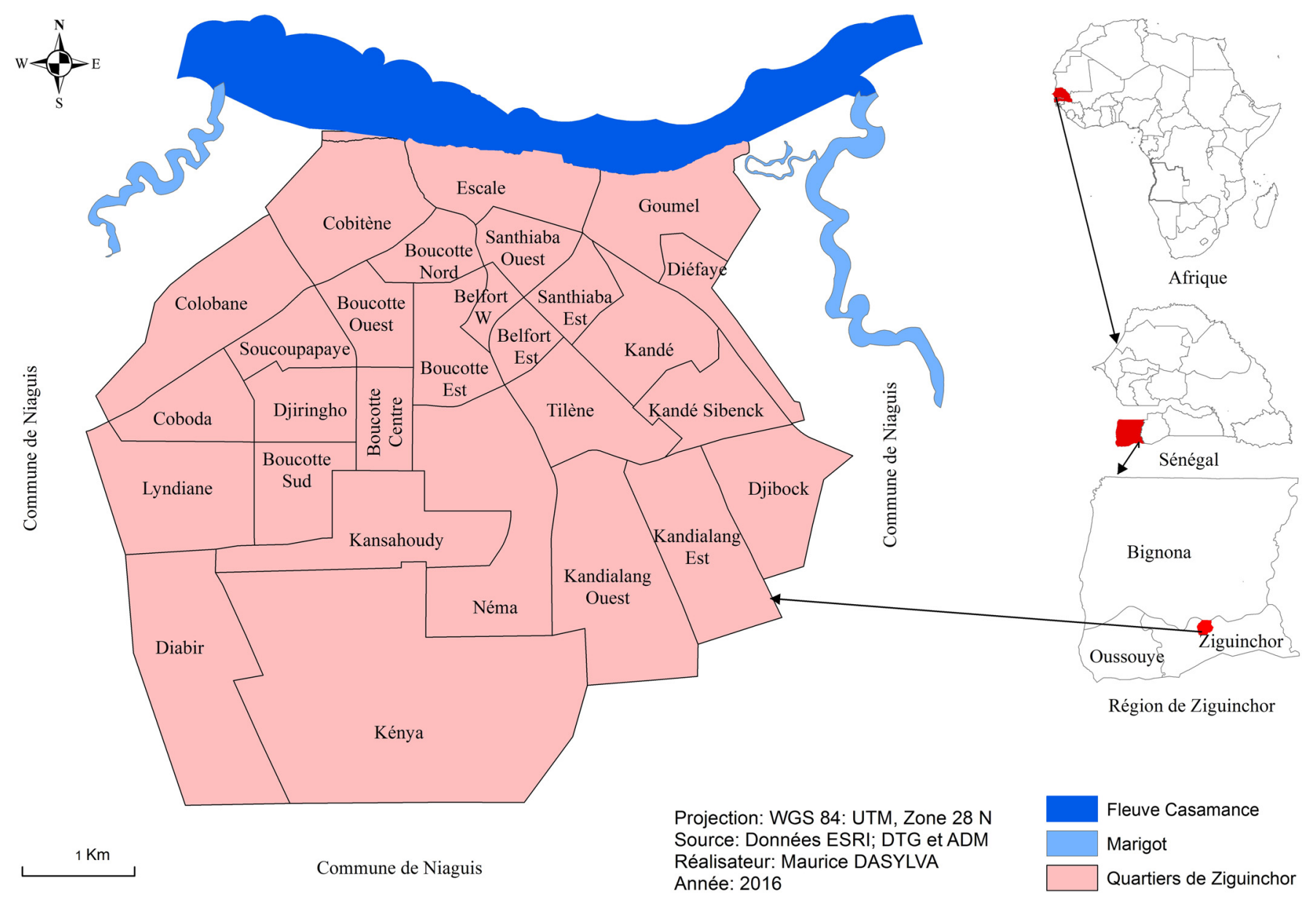

Fig. 1. Carte de localisation de Ziguinchor.

Fig. 1. Location map of Ziguinchor.

consisté à repérer des parcelles de PAM et à identifier ensuite de proche en proche leurs exploitants. Les levées de surface et la prise des coordonnées géographiques des parcelles ont été réalisées à l'aide d'un GPS.

Pour l'enquête, nous avons établi un questionnaire qui porte sur les caractéristiques des ménages des producteurs, les pratique de culture, le statut et la tenure du foncier exploité, les services environnementaux et socioéconomiques des microexploitations des PAM. Il a servi à collecter les données auprès de 70 producteurs répartis dans 5 quartiers.

Les différentes espèces de menthe cultivées ont été identifiées en collaboration avec les producteurs et à l'aide de la flore illustrée de Berhaut (1988) pour ce qui relève de la systématique.

\subsection{Le traitement des données}

Les données cartographiques ont été corrigées et extraites par le logiciel MapSource sous format de fichier gpx. Elles ont été vectorisées à l'aide du logiciel Qsis. Les cartes ont été réalisées l'aide du logiciel ArcGIS 10.2.2.

Les données issues des enquêtes ont été traitées à l'aide du logiciel Sphinx Plus ${ }^{2}$. Il a permis de réaliser des analyses univariées portant sur les micro-exploitations de PAM. Elles ont permis de déterminer les tendances centrales des différentes variables.

\section{Résultats}

Les résultats de l'étude décrivent les caractéristiques des micro-exploitations des PAM et leurs fonctions environnementales et socioéconomiques dans le contexte spécifique de l'agriculture urbaine à Ziguinchor.

\subsection{Caractéristiques des micro-exploitations de plantes aromatiques et médicinales}

\subsubsection{Plantes, lieux et techniques de production}

Les PAM cultivées à Ziguinchor sont composées de trois espèces de la famille des Lamiaceae (Fig. 2) : deux espèces du genre Mentha, dont la menthe poivrée (Mentha X piperita L.) et la menthe verte (Mentha spicata L.), et le basilic (Ocimum basilicum L.). Ces cultures sont respectivement appelées, dans la langue locale, «Nanah menthe», «Nanah ordinaire» et «Nanah fass ». La menthe verte est la plus cultivée selon $42 \%$ des personnes interrogées. Elle est suivie par la menthe poivrée et le basilic qui représentent, respectivement, $35 \%$ et $23 \%$ des citations. 


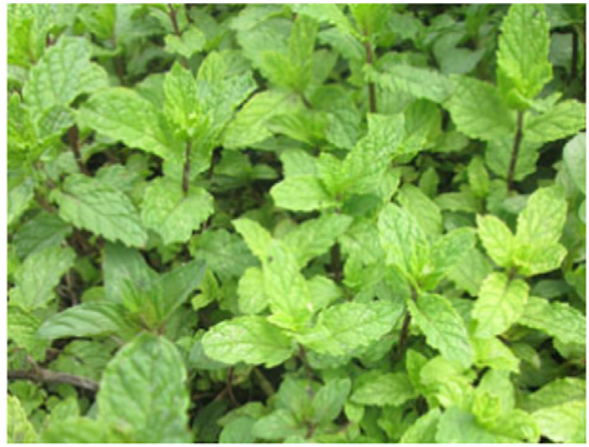

Mentha x piperita L.

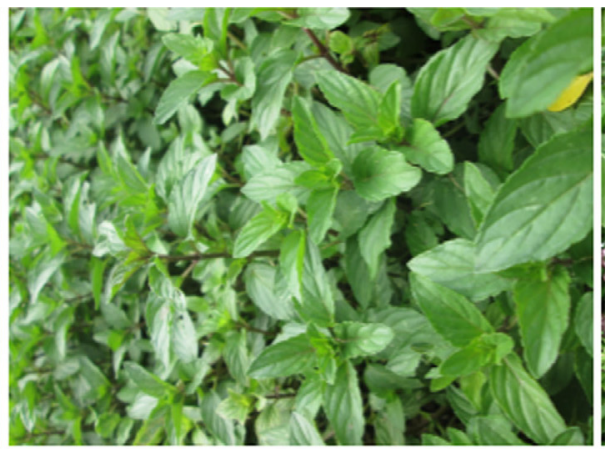

Mentha spicata L.

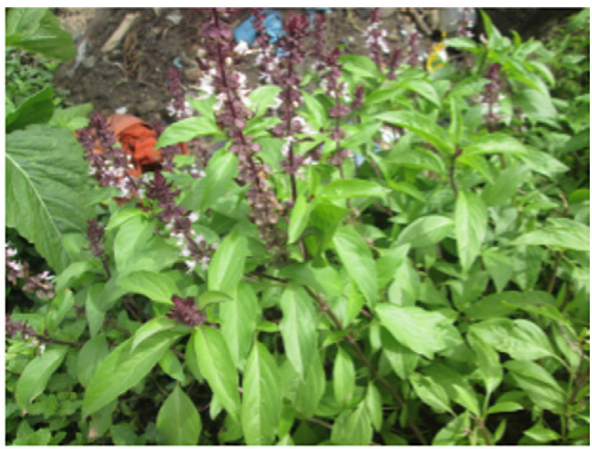

Occimum basilicum L.

Fig. 2. Plantes aromatiques cultivées à Ziguinchor.

Fig. 2. Aromatic plants cultivated in Ziguinchor.

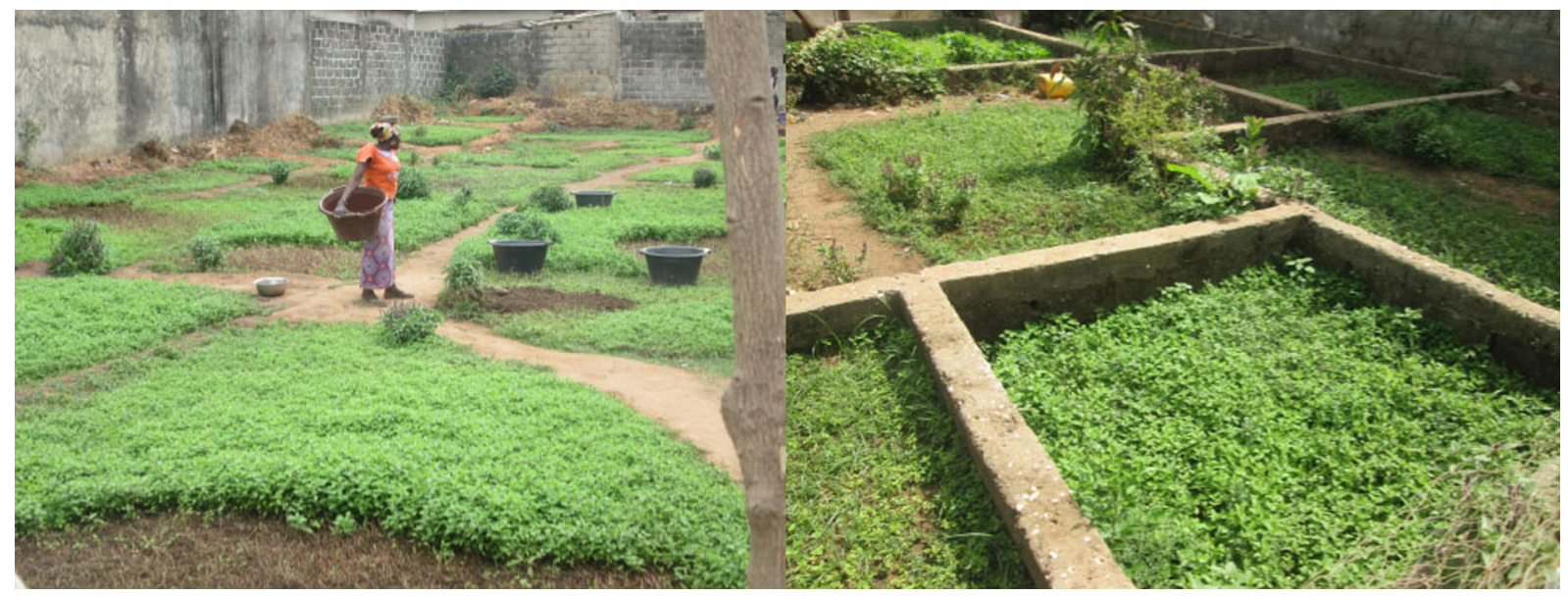

Fig. 3. Cultures de menthe dans les maisons et bâtiments en construction.

Fig. 3. Mint production in settlements and buildings under construction.

La production est réalisée en milieu urbain. Quarante-trois pour cent des producteurs installent leurs exploitations dans des parcelles vacantes à usage d'habitat, $34 \%$ exploitent les bordures des routes non bitumées et $28 \%$ cultivent dans les habitations (Fig. 3). La taille de ces micro-exploitations varie entre 7 et $50 \mathrm{~m}^{2}$. Un producteur cultive en moyenne $28,4 \mathrm{~m}^{2}$ par an. Les quartiers de Nema et Kandialang Est concentrent près de $85 \%$ des exploitations répertoriées (Fig. 4). Ils sont majoritairement peuplés par la communauté mandingue, qui s'est spécialisée dans cette production.

Les PAM sont majoritairement produites en contre-saison par $56 \%$ des producteurs. Les autres $(44 \%)$ les cultivent pendant la saison des pluies. Les micro-exploitations sont installées uniquement en pleine terre et la main-d'œuvre est familiale. La préparation du lit de semence est essentiellement réalisée à l'aide du «fantingho», un instrument rudimentaire de labour jadis utilisé par les femmes mandingues dans la production rizicole. Le sol labouré est fumé et arrosé pendant quelques jours avant la mise en terre des cultures. Le mode de multiplication des menthes est essentiellement végétatif, par bouturage. Les boutures sont repiquées en ligne de poquets équidistants de 20 à $30 \mathrm{~cm}$.
Les menthes, cultures exigeantes en eau, sont irriguées en contre-saison uniquement avec de l'eau des puits. Les producteurs utilisent environ 10 à 15 bassines d'eau de 20 litres par jour pour irriguer des parcelles de 10 à $15 \mathrm{~m}^{2}$. Pendant la saison des pluies, la fréquence d'irrigation et la quantité d'eau utilisée diminuent considérablement. Toutefois, les producteurs arrosent leurs parcelles avec l'eau des puits après chaque pluie.

Les sols sont principalement fertilisés avec de la matière organique. Les ordures ménagères, la fumure animale et les engrais minéraux (NPK et urée) sont épandus sur respectivement $55 \%, 24 \%$ et $21 \%$ des parcelles fertilisées. À maturité, les menthes présentent un bon développement de leurs organes végétatifs. Les parcelles sont alors récoltées par rotation, journellement ou tous les deux jours. Cette technique permet aux producteurs d'étaler la récolte dans le temps et de minimiser les pertes post-récolte liées à la mévente.

\subsubsection{Tenure foncière et profil socioprofessionnel des productrices}

La tenure foncière des micro-exploitations de PAM relève, dans $83 \%$ des cas, de l'emprunt gratuit de terres par consensus 


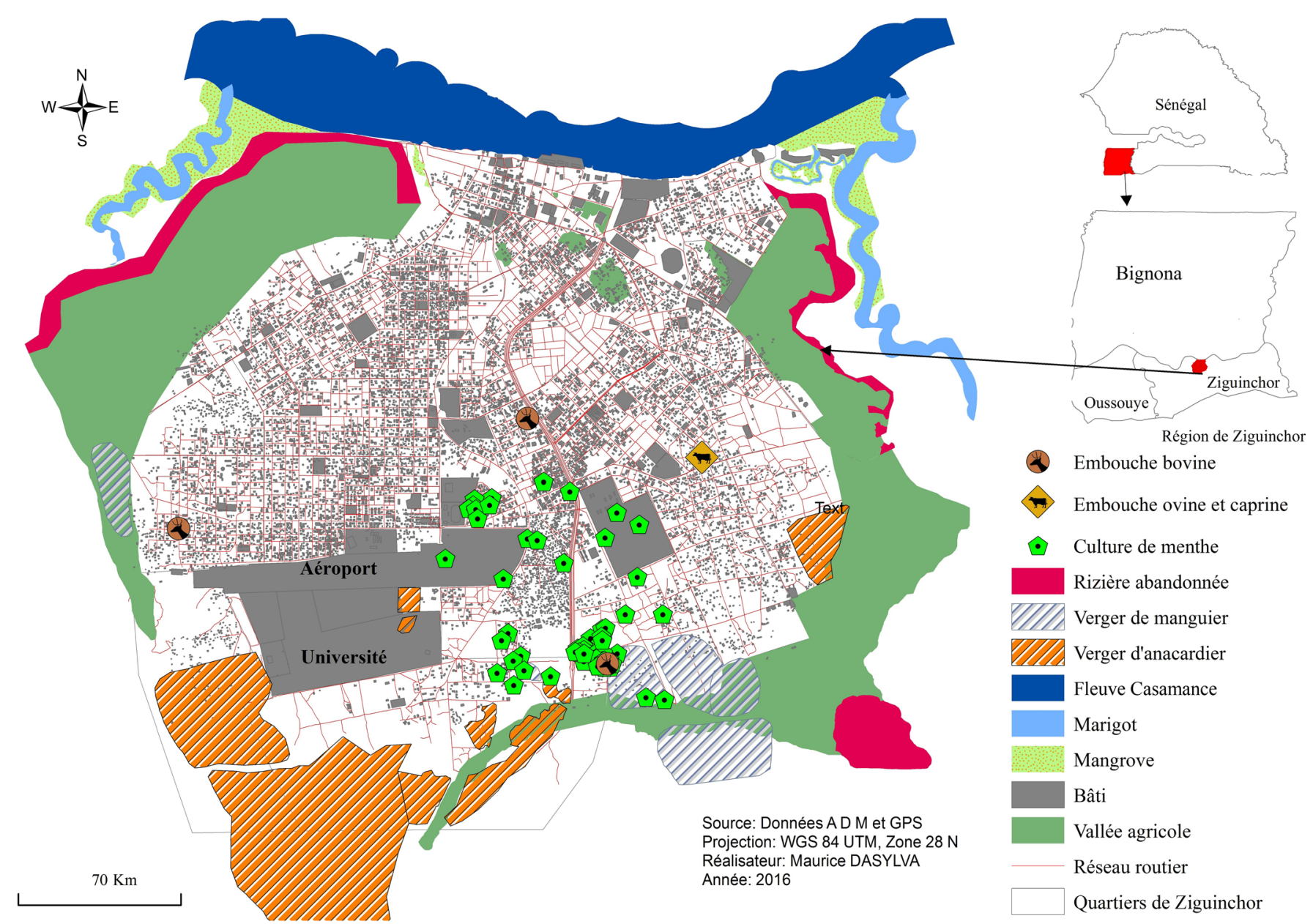

Fig. 4. Occupation des sols et micro-exploitations de menthe étudiées à Ziguinchor.

Fig. 4. Land use and micro-farms of mint surveyed in Ziguinchor.

entre les propriétaires et les producteurs. Dans ce type de contrat oral ne requérant aucune condition financière, le bénéficiaire est tenu de renouveler annuellement sa demande d'exploitation de la parcelle. Le propriétaire terrien ne contrôle pas l'effectivité des pratiques sur la parcelle. Dans $8 \%$ des cas, les producteurs sont les propriétaires légaux de leurs parcelles; $6 \%$ les louent pour 11000 francs CFA/an en moyenne et $3 \%$ exploitent des parcelles sises dans les espaces publics. Il s'agit, dans ce dernier cas de figure, des bordures des routes et boulevards non bitumés.

La production des PAM est essentiellement effectuée par des femmes de 17 à 65 ans, qui détiennent $99 \%$ des microexploitations. Du point de vue matrimonial, $81 \%$ des productrices sont mariées et $13 \%$ sont veuves. Elles vivent dans des ménages composés en moyenne de 16 personnes. Soixantetreize pour cent d'entre elles sont analphabètes. Seules $16 \%$ ont un niveau de scolarité primaire et $11 \%$ ont étudié au niveau secondaire. Parmi les familles rencontrées, $10 \%$ des productrices sont des déplacées du conflit casamançais. L'agriculture représente le principal moyen de subsistance de ces productrices, puisque la culture des PAM représente $86 \%$ de leurs activités agricoles en termes de temps actif. Les autres activités agricoles sont l'élevage (14\%), les grandes cultures (riz, mils, maïs, arachide) et l'arboriculture fruitière.

\subsubsection{Facteurs limitant le développement des micro- exploitations}

Plusieurs facteurs limitent le développement des microexploitations. La tenure foncière est basée sur le prêt de terres, sur la production en pleine terre et sur l'occupation de parcelles à usage d'habitat, ce qui est source de précarité. L'urbanisation de ces parcelles à usage d'habitat se profile à court terme, compte tenu de la spéculation foncière qui sévit en ville. Dans ce contexte, la mairie et le service d'urbanisation sont responsables des attributions foncières et des délivrances d'autorisations de construction. Dépassées par l'urbanisation galopante, ces institutions ne considèrent pas la mise en culture d'une parcelle comme un frein à la délivrance d'un permis de construire. Les producteurs sont parfois délogés de leurs exploitations pour faire place à la construction de nouveaux bâtis, comme l'attestent $34 \%$ des personnes interrogées. À cela s'ajoutent les dégâts dus à la divagation du bétail, la perte des semis due aux attaques fongiques, la prédation des plantes par les bio-agresseurs aériens, le manque d'eau d'irrigation et les pertes post-récolte. Tous ces facteurs limitent la productivité de ces micro-exploitations. Mais malgré les facteurs de vulnérabilité qui pèsent sur elle, la production des PAM joue un rôle indispensable du point de vue environnemental et socioéconomique dans la commune. 


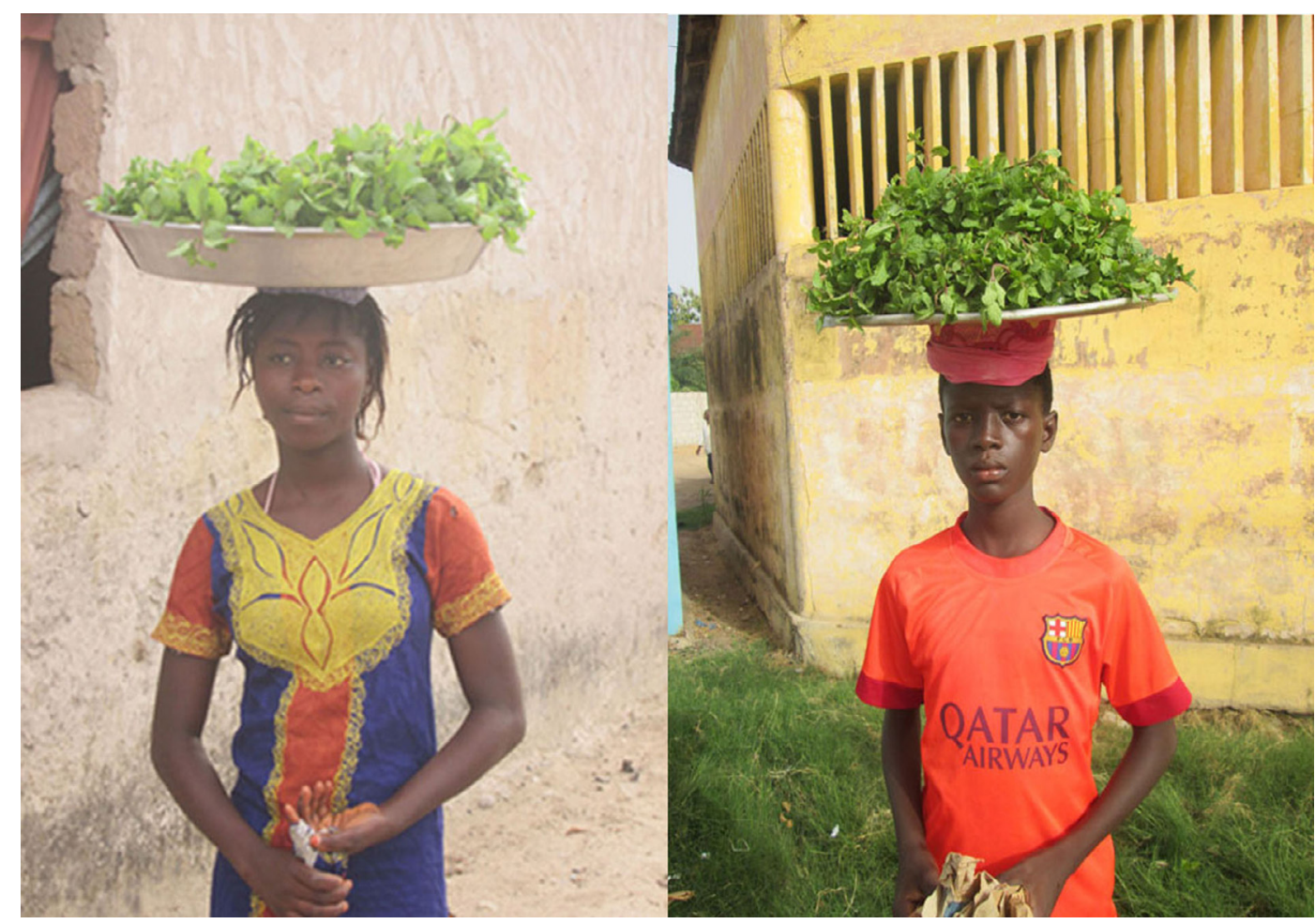

Fig. 5. Vendeurs ambulants de plantes aromatiques et médicinales dans le quartier de Néma 2.

Fig. 5. Street traders of aromatic and medicinal plants in Nema 2 district.

\subsection{Fonctions environnementales et socioéconomiques}

\subsubsection{Fonctions environnementales}

La production des PAM, à l'instar des autres types de cultures, contribue au fonctionnement du système de collecte des ordures ménagères urbaines, utilisées pour fertiliser les sols. Quatre-vingt-neuf pour cent des producteurs déclarent utiliser ces ordures comme fertilisants. Soixante et onze pour cent de ces ordures proviennent des ménages eux-mêmes, le reste est collecté dans les poubelles des quartiers. À ces ressources s'ajoute la collecte de la fumure animale réalisée auprès des éleveurs. Il faut noter, par conséquent, la capacité de ce système d'exploitation agricole à assainir la ville et à améliorer son cadre de vie. La fonction d'assainissement urbain liée à ce système est d'autant plus importante que les $73 \%$ des personnes interrogées déclarent que les éboueurs de la municipalité ne gèrent que les déchets du centre urbain. Ces micro-exploitations sont donc un facteur de santé publique à Ziguinchor.

\subsubsection{Fonctions socioéconomiques}

La fonction socioéconomique des micro-exploitations de PAM repose essentiellement sur leur capacité à générer des revenus pour les producteurs. La vente est réalisée par les enfants de 7 à 15 ans et de manière ambulante à travers la ville (Fig. 5). Parmi ces enfants, $68 \%$ sont des filles.

Dans le quartier de Néma 2, $84 \%$ de la production est vendue par les enfants, à raison de 25 FCFA la botte de feuilles fraîches $(1 €=656$ FCFA $)$. Les revenus moyens annuels par producteur sont évalués à 207462 FCFA (soit $43 \%$ de celui d'un ménage agricole de Ziguinchor). Ils peuvent atteindre 960000 FCFA dans certains cas. Soixante-deux pour cent de ces revenus servent à l'achat de nourriture, aux frais de scolarité et aux besoins vestimentaires. L'autre partie des revenus (38\%) est destinée aux frais sanitaires des ménages, au loyer ou aux factures d'électricité.

L'importance socioéconomique des menthes est aussi illustrée par une productrice du quartier de Néma 2 qui obtient des résultats spectaculaires. Une veuve, outre l'assurance des besoins substantiels de son ménage, a payé son terrain et construit sa maison grâce aux revenus qu'elle tire de la culture des menthes. D'ailleurs, certaines femmes affirment que cette activité leur procure un statut social. En effet, dans un contexte de pauvreté et de faible pouvoir d'achat des ménages, les femmes aident leur mari à supporter les charges financières du foyer.

\section{Discussion}

Cette étude a permis de caractériser les micro-exploitations de PAM et d'apprécier leurs fonctions environnementales et socioéconomiques à Ziguinchor.

\subsection{Vulnérabilité des micro-exploitations}

L'insécurité foncière constitue le principal facteur limitant de la production des PAM dans la ville. Un producteur exploite en moyenne $28,4 \mathrm{~m}^{2}$ par an. La taille des parcelles cultivées est très faible, comparée à la taille moyenne des exploitations de la province de Settat (Maroc), où un producteur exploite environ 0,1 à 0,5 ha de menthe par an, voire 2 à 6 ha dans certains cas 
(Ministère de l'agriculture et de la pêche maritime [MAPM], 2008). D'ailleurs, le recours à l'emprunt gratuit de terres dans $83 \%$ des cas ne permet pas aux producteurs de Ziguinchor d'exercer leur activité pendant plus d'un an dans une même parcelle, en raison de l'expropriation fréquente (fin de contrat ou construction de nouveaux bâtis). Compte tenu de la problématique du foncier dans cette ville, les techniques de production hors-sol, développées sur les balcons, les terrasses, et dans les cours des habitats, pourraient contribuer à la durabilité du système d'exploitation pratiqué jusque-là en pleine terre.

À Ziguinchor, l'irrigation des micro-exploitations de PAM est basée sur l'utilisation de l'eau des puits. Cette technique d'irrigation fastidieuse nécessite beaucoup d'énergie pour remonter chaque jour 0,2 à $0,3 \mathrm{~m}^{3}$ d'eau d'une profondeur de $10 \mathrm{~m}$ en moyenne. L'obligation d'arroser les cultures de menthes après chaque pluie tient à la nécessité de rincer l'eau de pluie imbibant les feuilles des cultures. Selon les producteurs, l'eau de pluie favorise les attaques et infections des plants, alors que l'eau du robinet et les engrais minéraux altèrent leurs qualités organoleptiques et aromatiques, en plus du blocage de leur croissance végétative. Ce constat des producteurs pourrait être lié à la présence de produits chimiques utilisés (chlore) pour désinfecter l'eau de la Sénégalaise des eaux (SDE). Ces produits peuvent être nuisibles à la physiologie des cultures.

À Ziguinchor, $80 \%$ des producteurs utilisent les ordures ménagères et de la bouse animale comme fertilisants dans les exploitations. Ils font remarquer que les fertilisants organiques favorisent le développement végétatif des cultures. Ce résultat corrobore ceux de Nouatin et Bachabi (2010), qui précisent que les maraîchers de la ville de Parakou au Bénin utilisent essentiellement les fertilisants organiques pour stimuler et maintenir, de façon durable, la productivité de leurs exploitations. D'ailleurs, ce système d'exploitation est peu consommateur de produits chimiques à Ziguinchor, comparé à l'agriculture urbaine à Dakar, où près de 62 produits chimiques utilisés ont été identifiés par Fall et al. (2006).

\subsection{Effet du système sur l'environnement et la pauvreté}

La production des PAM évite la prolifération des ordures et assainit la ville selon $87 \%$ des personnes interrogées. En effet, les ordures ménagères et les débris végétaux collectés sont brûlés ou compostés. Les cendres ou l'humus obtenus sont utilisés pour fertiliser les sols. Quatre-vingt-neuf pour cent des producteurs utilisent les déchets urbains organiques comme fertilisants, $71 \%$ provenant des ménages et $29 \%$ des poubelles. Certains producteurs nettoient et occupent les dépotoirs irréguliers pour y installer leurs exploitations. Le terreau des dépotoirs riche en humus permet de maximiser leur production. L'agriculture urbaine est donc un secteur palliatif de la gestion des déchets, dans un contexte où le service d'assainissement n'assure que $50 \%$ du ramassage (Nyassogbo, 2004). Ce mode de fertilisation des sols contribue aussi à réduire le risque d'apparition de maladies pulmonaires qui pourraient être causées par l'incinération des déchets solides (Sall et Sy, 2015). Il a des effets bénéfiques sur la santé publique. Selon $80 \%$ des producteurs interrogés eux-mêmes, l'agriculture contribue à l'amélioration du cadre de vie et de la santé des populations.

Outre sa capacité à contribuer à l'assainissement urbain, la production des menthes joue un rôle indispensable du point de vue socioéconomique dans la commune. La production vendue est source de revenus pour les producteurs. Ces revenus, relativement faibles, jouent un rôle d'appoint en complément de la production autoconsommée des autres cultures. Ils servent à la scolarisation des enfants pour $62 \%$ des répondants. Ces résultats corroborent ceux de Munkuamo et al. (2015), selon qui les revenus du maraîchage permettent à $68 \%$ des producteurs d'envoyer leurs enfants à l'école à Kinshasa, en République démocratique du Congo. D'ailleurs, $10 \%$ des producteurs des PAM de Ziguinchor sont des déplacés du conflit casamançais. Ce sont eux qui ont les plus grandes difficultés d'accès au foncier, à l'instar des agriculteurs urbains de Dar es Salaam en Tanzanie (McLees, 2011). De plus, ils n'ont que ce système de production pour assurer leurs besoins de subsistance. Ces déplacés, peu scolarisés et sans qualification professionnelle pour $90 \%$ d'entre eux, comptent sur la culture des PAM pour lutter contre une situation de pauvreté.

Les micro-exploitations de PAM contribuent aussi au renforcement de la cohésion sociale, ce que soulignent $91 \%$ des personnes interrogées. En effet, les productrices s'organisent en groupements d'intérêt socioéconomique où chaque membre verse une cotisation hebdomadaire, mensuelle, ou bimensuelle. Lors d'une tontine, le choix du bénéficiaire se fait par tirage au hasard sans remise. Selon les circonstances, elles organisent des cotisations pour venir en aide à un membre nécessiteux (décès, mariage ou baptême). Il s'y ajoute la stabilité des ménages, soulignée par les productrices qui soutiennent leur mari avec les revenus tirés de la menthe.

Cependant, la vente ambulante des menthes par des enfants de 7 à 15 ans pose un problème de sécurité. Les filles sont ainsi exposées aux viols et grossesses précoces. Cependant, les parents déclarent leur impuissance en raison de l'importance des revenus des PAM dans la subsistance des ménages. Par ailleurs, cette activité provoque le décrochage scolaire des enfants, comme le soulignent certaines productrices. Pour y pallier, certaines engagent des talibés (garçons confiés aux marabouts qui sont chargés de leur transmettre le savoir coranique) pour la vente des produits. En contrepartie, les talibés reçoivent de la nourriture et la rétribution journalière que leurs maîtres coraniques exigent d'eux.

\section{Conclusion}

Cette étude a permis de caractériser les micro-exploitations des PAM et de tenter d'apprécier leurs fonctions environnementales et socioéconomiques à Ziguinchor. La menthe verte, la menthe poivrée et le basilic sont des espèces cultivées en ville par les femmes. Ces micro-exploitations contribuent à l'assainissement urbain utilisant des ordures ménagères pour la fertilisation des sols. La production vendue contribue pour une part significative aux moyens d'existence des ménages. Si les revenus moyens annuels, évalués à 207462 FCFA, sont relativement faibles, ils jouent un rôle d'appoint à la production autoconsommée des autres cultures.

Cependant, cette activité peine à se développer à cause de diverses contraintes qui pèsent sur elle, notamment la précarité 
foncière et l'exposition à la divagation du bétail. Des solutions pourraient être promues pour réduire cette précarité foncière, en favorisant par exemple des modes de production hors-sol (micro-jardinage), développés sur les balcons, les terrasses et dans les cours des maisons.

Cette production pourrait également trouver d'autres débouchés économiques. En effet, bon nombre d'usages et vertus des menthes ne sont pas valorisés. On peut souligner, à titre d'exemple, le caractère sensible des souches de bactéries du genre Staphylococcus à l'effet synergique de l'huile d'argan avec celui de la menthe poivrée (Lotfi et al., 2015), ainsi que la capacité de cette menthe à soigner les troubles digestifs et respiratoires (Debuigne et Couplan, 2009; Pouzergues, 2013). Toutes ces vertus des menthes sont méconnues des producteurs et des consommateurs locaux.

Au-delà du cas local de Ziguinchor, les micro-exploitations de PAM sont un cas exemplaire des micro-activités agricoles en ville qui procurent un «mieux-vivre» à des populations citadines en situation de pauvreté. Si elles connaissent des limites à leur développement, des solutions d'amélioration existent, qui pourraient être mises en œuvre à moindre coût.

\section{Références}

Akinbamijo O, Fall ST, Smith OB. 2002. Advances in crop-livestock integration in West Africa cities. The Netherlands: Grafisch Bedrijf ponsen and looijen, 214 p. ISBN: 90-6464-982-0.

ANSD. 2014. Rapport définitif RGPHAE 2013. Chapitre X, Le ménage, $20 \mathrm{p}$.

ANSD. 2015. Rapport de projection de la population du Sénégal: 2013-2063, $175 \mathrm{p}$

Ba A, Cantoreggi N, Simos J, Duchemin E. 2016. Impacts sur la santé des pratiques des agriculteurs urbains à Dakar (Sénégal). VertigO la revue électronique en sciences de l'environnement 16(1): $16 \mathrm{p}$. DOI: $10.4000 /$ vertigo. 17030 .

De Bon H, Parrot L, Moustier P. 2010. Sustainable urban agriculture in developing countries. A review. Agron Sustain Dev 30(1): 2132. DOI: 10.1051 /agro:2008062.

Berhaut J. 1988. Flore illustrée du Sénégal. Dakar : Clairafrique, Tome I-IX.

Dasylva M. 2013. Agriculture urbaine sous pluie et sécurité alimentaire dans la commune de Ziguinchor. Mémoire de Master. Ziguinchor, Sénégal : AGDEFA, UF-ST, Université Assane Seck de Ziguinchor, $53 \mathrm{p}$.

Debuigne G, Couplan F. 2009. Petit Larousse des plantes médicinales. Paris: Larousse, Collection Nature et Jardin, 383 p. ISBN: 978-2035838810.

Dufour C, Larivière V. 2012. Principales techniques d'échantillonnage probabilistes et non-probabilistes, SCI6060 - Cours 4. [Consulté le 15/11/2017]. Disponible sur: http://cours.ebsi.umon treal.ca/sci6060/docs/sci6060_c4_fiche_echantillon.pdf.

Fall ST, Fall AS, Cissé I, Badiane A, Fall CA, Diao MB. 2006. Intégration horticulture-élevage dans les systèmes agricoles urbains de la zone des Niayes (Sénégal). Bulletin de l'APAD 19 | 2000. Disponible sur: http://apad.revues.org/444.

FAO. 2010. Agriculture intelligente face au climat, Food and Agriculture Organization. [Consulté le 03 janvier 2018]. http:// www.fao.org/climatechange/climatesmart/66250/fr/.

FAO. 2014. Growing greener cities in Latin America and the Caribbean. Report on urban and peri-urban agriculture in the region. Disponible sur: http://www.fao.org/3/a-i3696e/i3696e00.pdf.
Hamilton AJ, Burry K, Mok HF, Fiona Barker S, Grove JR, Williamson V. 2013. Give peas of chance. Urban agriculture in developing countries: a review. Agron Sustain Dev 34:45-73. DOI: 10.1007/s13593-013-0155-8.

Keita A. 2013. La mutation des terres agricoles autour de Ziguinchor. Mémoire de master. Dakar, Sénégal: ATDDL, FLSH; Université Cheikh Anta Diop de Dakar, 96 p.

Kherri A. 2014. Échantillonnage, Support pédagogique de cours $\mathrm{n}^{\mathrm{o}}$ 02. Alger: École des hautes études commerciales, 20 p. http:// www.sg-ehec.jimdo.com.

Lotfi N, Chahboun N, El Hartiti H, Kabouche Z, El M'Rabet M, Berrabeh M, et al. 2015. Study of the antibacterial effect of Argan oil from Bechar region of Algeria on hospital resistant strains. $J$ Mater Environ Sci 6(9): 2476-2482.

MAPM. 2008. Transfert de la technologie en agriculture. Diagnostic dans la province de Settat (Royaume du Maroc). Conduite technique de la menthe, Programme national de transfert de technologie en agriculture(PNTTA). Bulletin mensuel d'information et de liaison du PNTTA. DERD, BP 6598, Rabat, Maroc, 6 p.

McLees L. 2011. Access to land for urban farming in Dar es Salaam, Tanzania: Histories, benefits and insecure tenure. The Journal of Modern African Studies 49(4): 601-624. DOI: 10.1017/ S0022278x11000498.

Mok HF, Williamson V, Grove JR, Burry K, Fiona Barker S, Hamilton A. 2013. Strawberry fields forever? Urban agriculture in developed countries: a review. Agron Sustain Dev 34: 21-43. DOI: 10.1007/ s13593-013-0156-7.

Mougeot LJA. 2006. Growing better cities, urban agriculture for sustainable development. Ottawa, Canada: CRDI, 113 p. ISBN 1-55250-227-9. http://www.crdi.ca/Livres.

Munkuamo JR, Munzundu BA., Nsimanda CI, Musibono DE. 2015. Vulnérabilités environnementales et stratégies de survie urbaines en situation post-conflit en République démocratique du Congo. ISSR Journals 13(1): 63-67.

Nyassogbo GK. 2004. La problématique de la gestion des ordures à Lomé. Annales de l'université de Lomé, Série Lettres, tome XXIV. Lomé, Togo: Presses universitaires de Lomé, p. 3-25.

Nouatin G, Bachabi FX. 2010. Urbanisation et viabilité de l'activité maraîchère : cas d'une ville à statut particulier au Bénin (Parakou). VertigO - la revue électronique en sciences de l'environnement 10 (2): 13 p. DOI: $10.4000 /$ vertigo. 10038 .

Oladokoun W. 2013. L'inefficacité du modèle de gestion du foncier rural au Togo. Étude de cas des agglomérations rurales périurbaines d'Atakpamé. Revue de Géographie Tropicale et d'Environnement 1: $45-65$.

Ouédraogo D. 2012. Perception des risques et consentement à payer pour une amélioration de la qualité des eaux usées pour le maraîchage au Burkina Faso : évidences empiriques à Ouagadougou et Bobo-Dioulasso au Burkina Faso. Annales de l'Université de Ouagadougou, Série B, nº 47, p. 133-174.

PAM. 2012. Rapport d'évaluation de la sécurité alimentaire en milieu urbain, communes de Kaolack, Kolda, Tambacounda et Ziguinchor. Rapport national du Sénégal, 24 p.

Pouzergues M. 2013. La migraine et ses alternatives thérapeutiques, Thèse de doctorat en pharmacie, université de Lorraine, $124 \mathrm{p}$.

Robin N. 2006. Le déracinement des populations en Casamance. Revue Européenne des Migrations Internationales 22(1): 153-181.

Robineau O, Soulard CT. 2017. Comprendre la complexité des liens ville-agriculture : intérêt d'une approche par le système agri-urbain. Le cas de Bobo-Dioulasso, Afrique de l'Ouest. Natures Sciences Sociétés 25(1): 36-47.

Sagna P. 2005. Dynamique du climat et son évolution récente dans la partie ouest de l'Afrique occidentale, Thèse de doctorat d'État es 
lettres. Dakar, Sénégal: Université Cheikh Anta Diop de Dakar, Faculté des lettres et sciences humaines, Département de Géographie, 790 p.

Sall O, Sy O. 2015. Défaillance institutionnelle et volontarisme populaire: les stratégies palliatives de gestion des déchets domestiques dans les périphéries urbaines de Dakar et Ziguinchor, Sénégal. DOI: 10.13140/RG.2.1.4469.7764.
Smith OB, Moustier P, Mougeot LJA, Fall A. 2004. Développement durable de l'agriculture urbaine en Afrique francophone. Enjeux, concepts et méthodes. Montpellier, France: CIRAD; Ottawa, Canada: CRDI, 176 p. ISBN: 2-87614-551-0, ISBN: 1-55250-134-5.

Zhiri A, Baudoux D. 2005. Huiles essentielles chémotypées et leurs synergies. Luxembourg: Éditions Inspir. ISBN: 2-919905- 27-9.

Citation de l'article : Dasylva M, Ndour N, Sambou B, Soulard C-T. 2018. Les micro-exploitations agricoles de plantes aromatiques et médicinales : élément marquant de l'agriculture urbaine à Ziguinchor, Sénégal. Cah. Agric. 27: 25004. 